\title{
Evaluation of a Proprietary Slow-Release Oxytocin Formulation on Corpus Luteum Function in Mares
}

\section{Brendan Sarnecky}

Dirk K. Vanderwall, Holly M. Mason, Stephen M. Kirschner, Benson Ambrose, Theda L. Parker
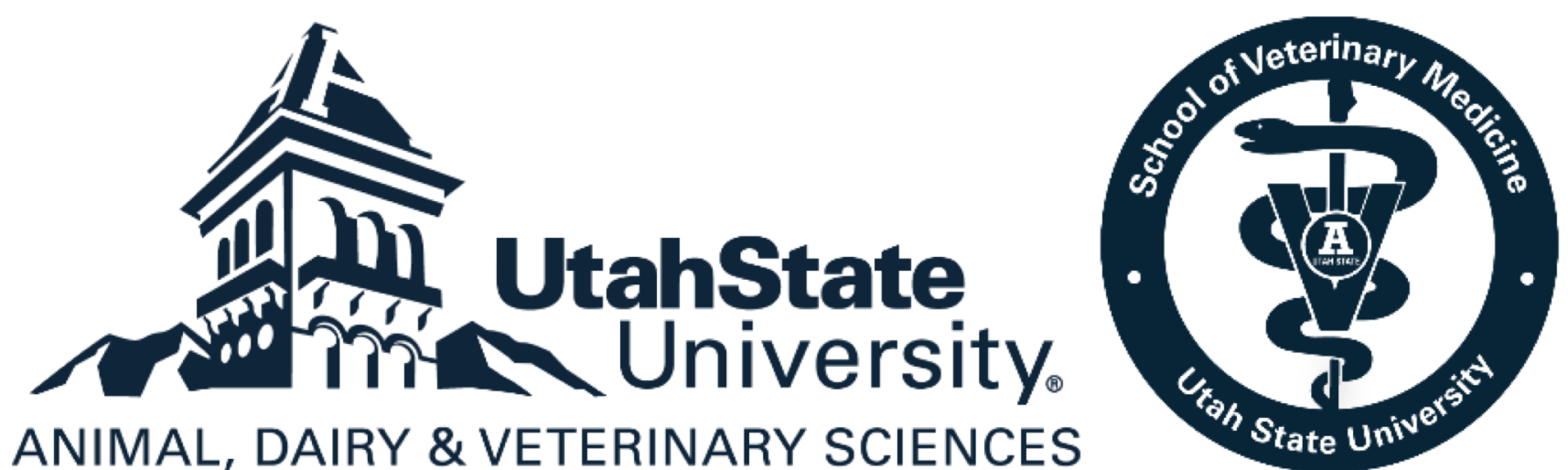


\section{Mare Estrous Cycle}

- 21 days

- 1 week estrus (i.e., "heat")

- Follicular phase

- Follicle- Estrogen

- 2 weeks diestrus

- Luteal phase

- Corpus Luteum (CL)- Progesterone

- Uterus

- Prostaglandin (PGF2 $\alpha$ )

- Measured as PGFM

- Luteolysis

- Posterior Pituitary- Oxytocin

- Role in luteolysis

\section{Equine Estrous Cycle}

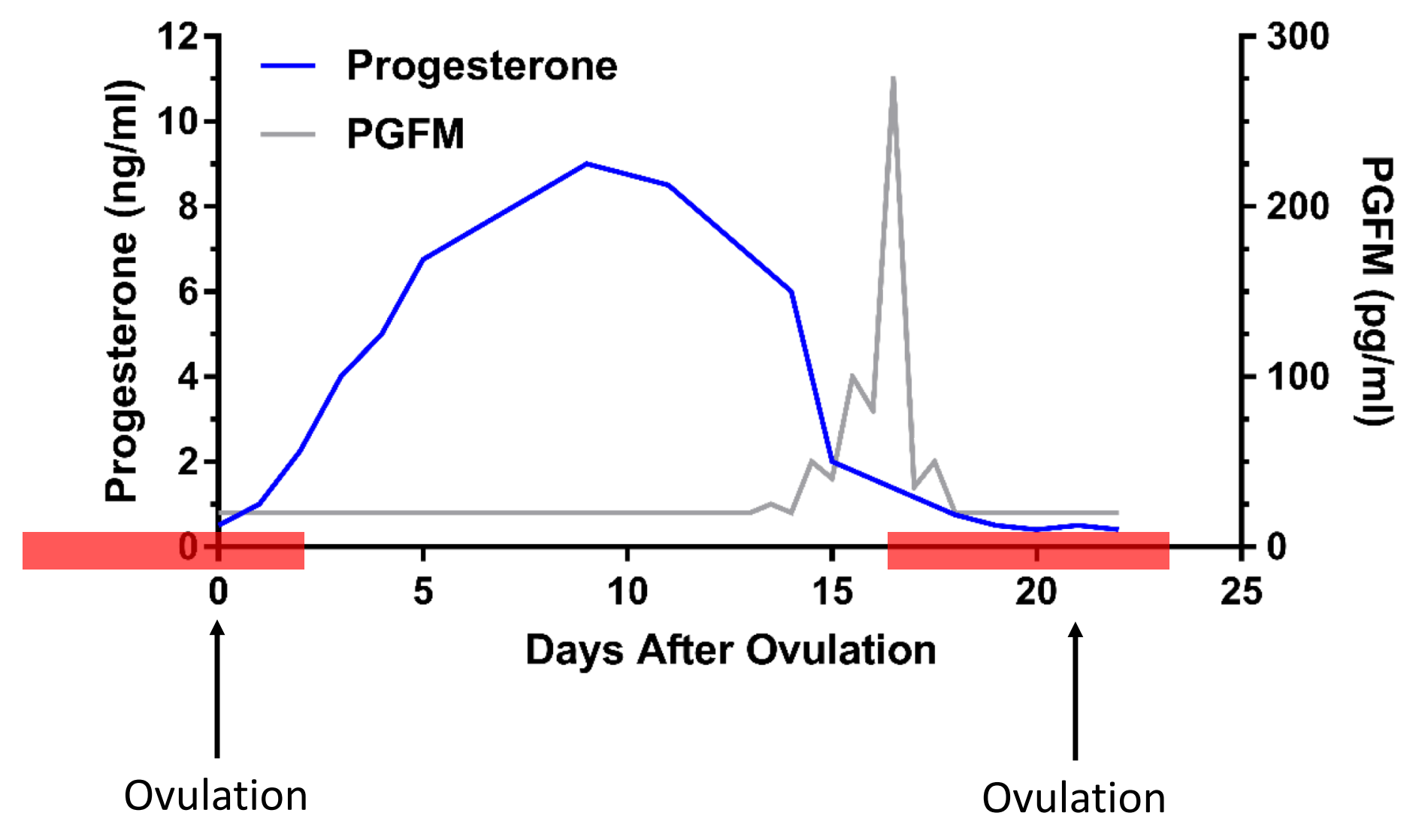




\section{Mare Estrous Cycle}

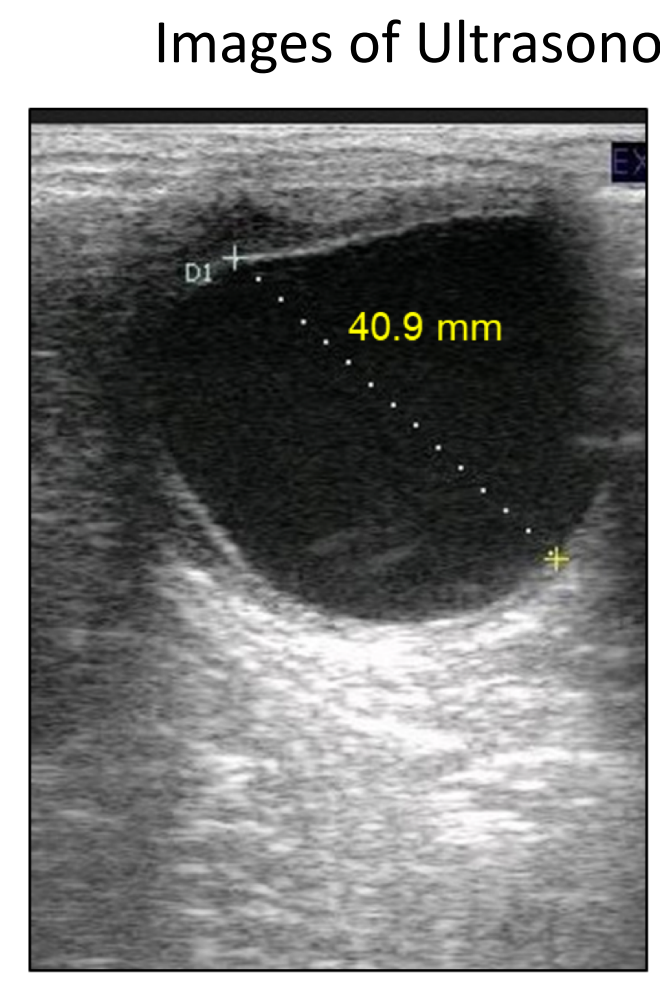

Follicle

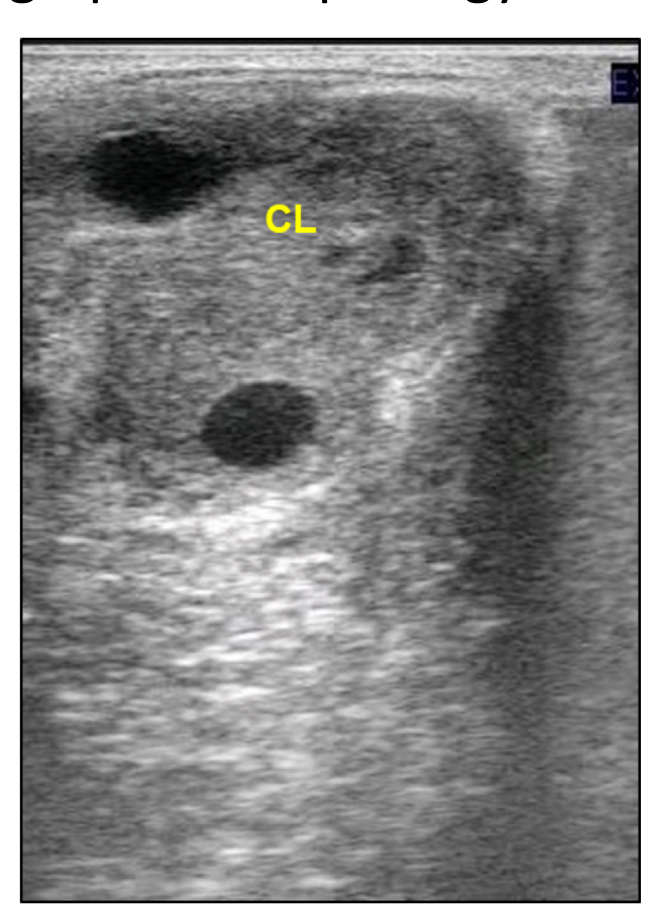

Corpus Luteum

\section{Equine Estrous Cycle}

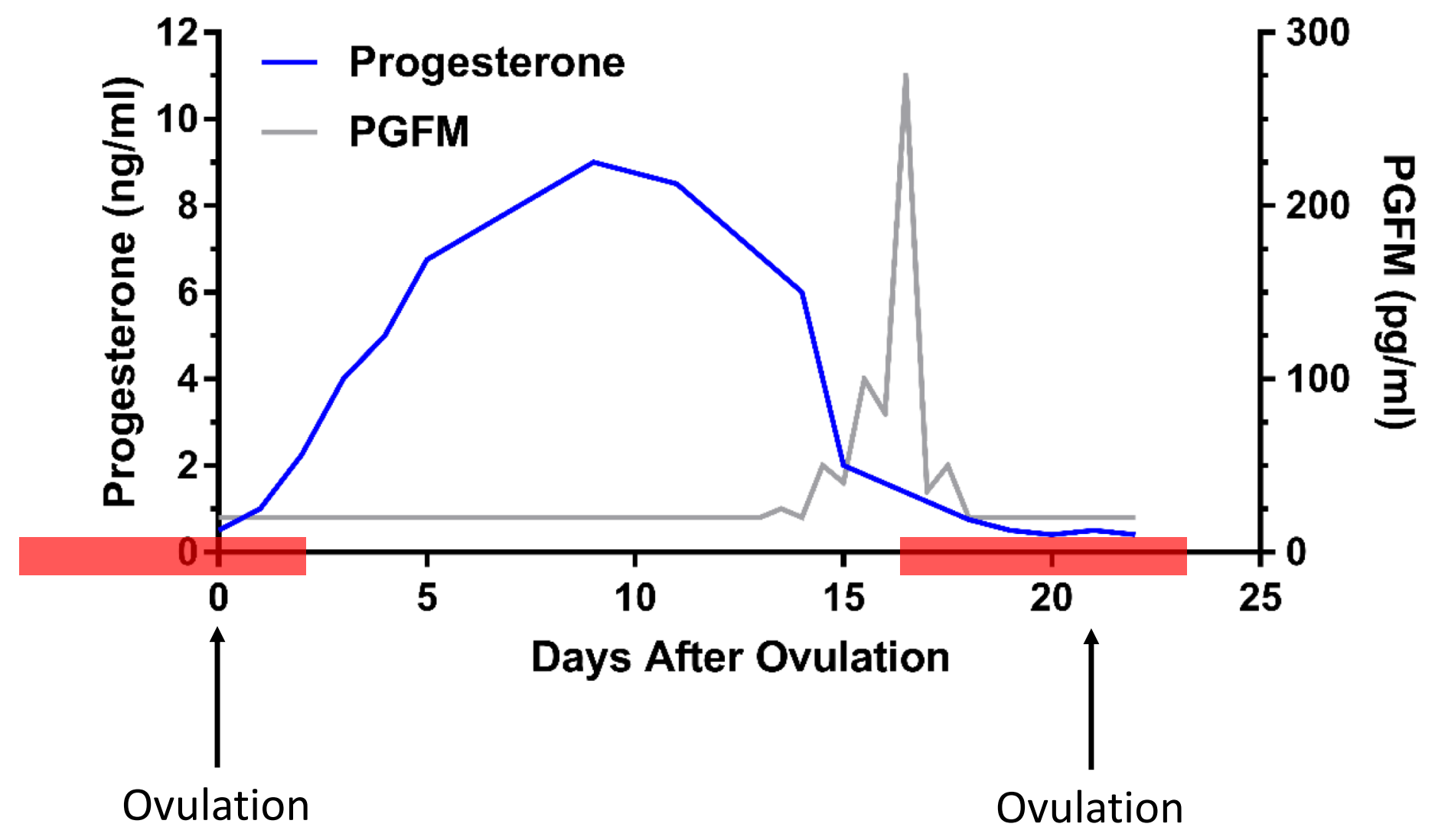




\section{Estrous Behavior (i.e., in heat)}
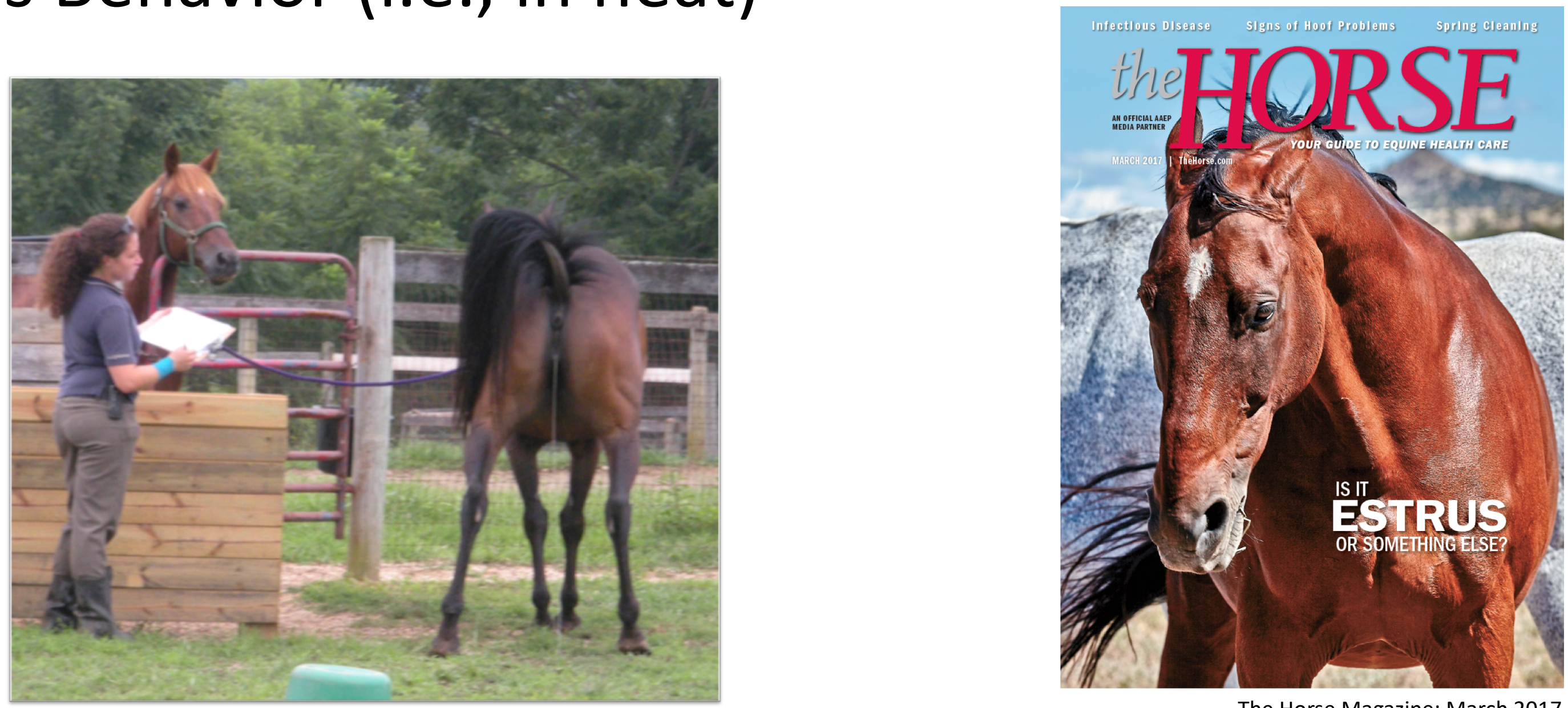

Courtesy of Dr. Vanderwall 


\section{Estrous Behavior (i.e., in heat)}

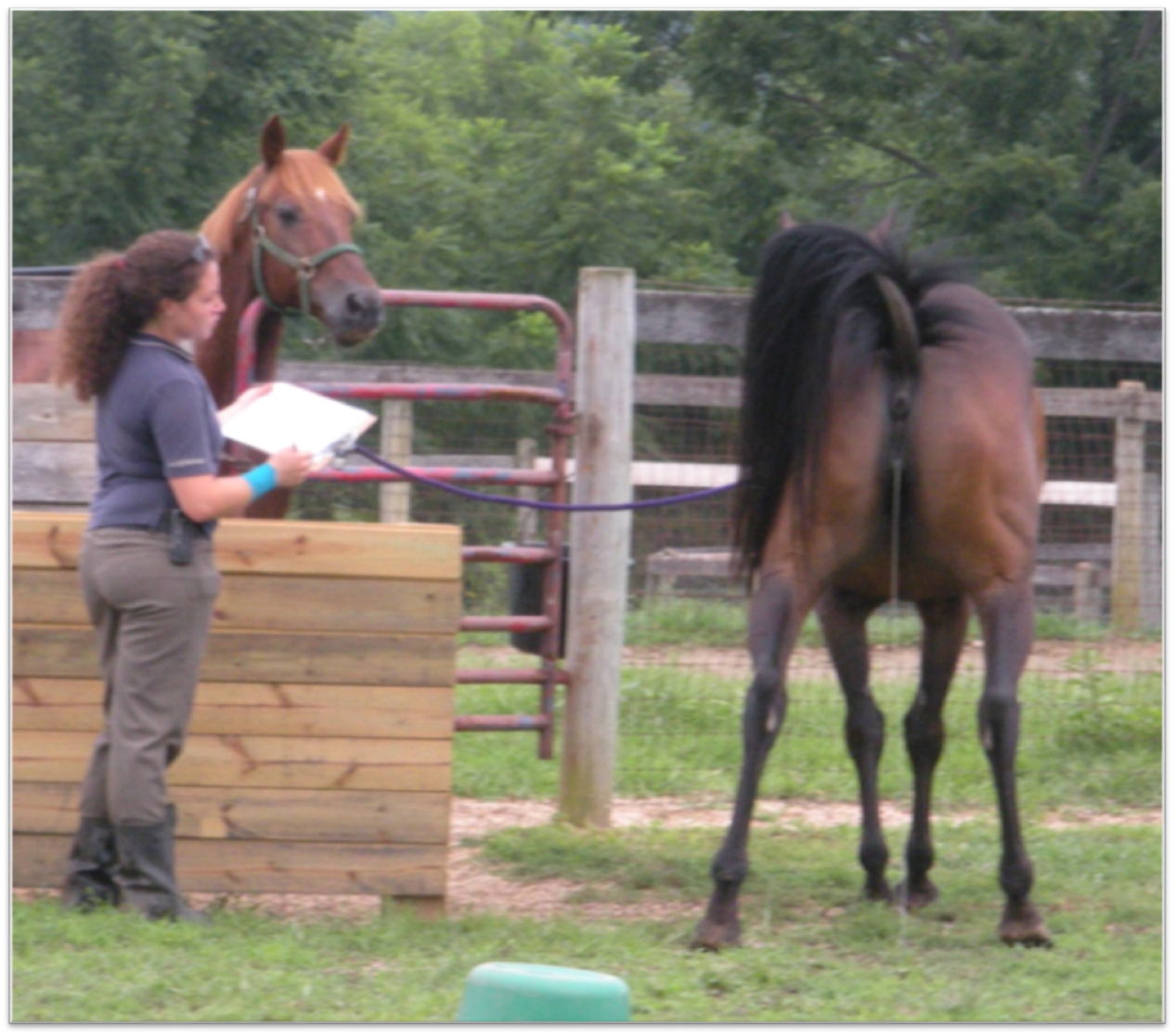

Courtesy of Dr. Vanderwall

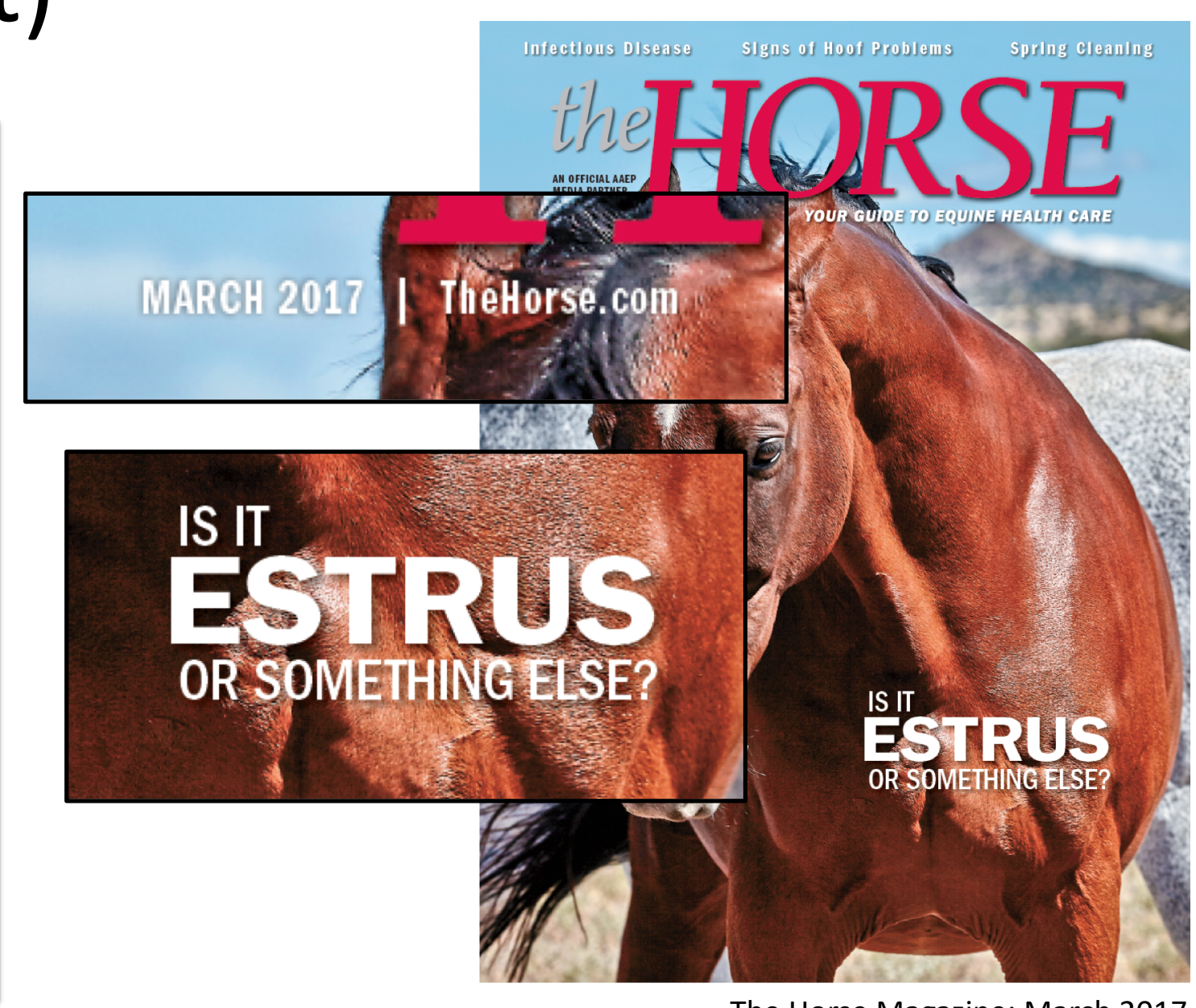

The Horse Magazine: March 2017 


\section{Estrus Suppression in the Performance Mare}
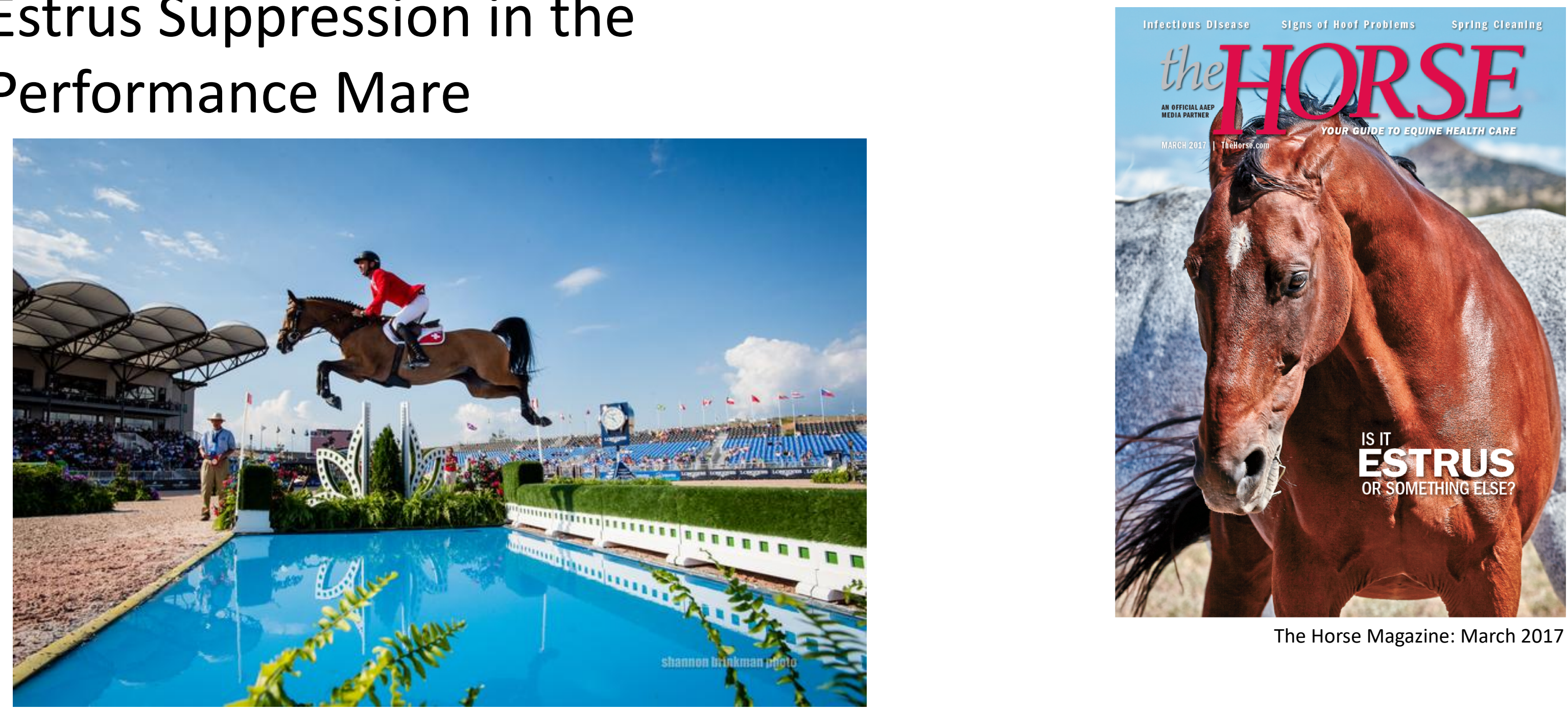

https://cdn.shopify.com/s/filies/1/0002/0899/4306/articles/GuerdatSteveSUI-WEG18brinkmang-19jRE2-
9561__20X.jpg 


\section{Methods of Estrus Suppression}

- Administration of exogenous progesterone/progestins

- E.g., Oral Altrenogest

- Extending the functional span of the corpus luteum $(\mathrm{CL})$

- Intrauterine glass ball

- Oxytocin

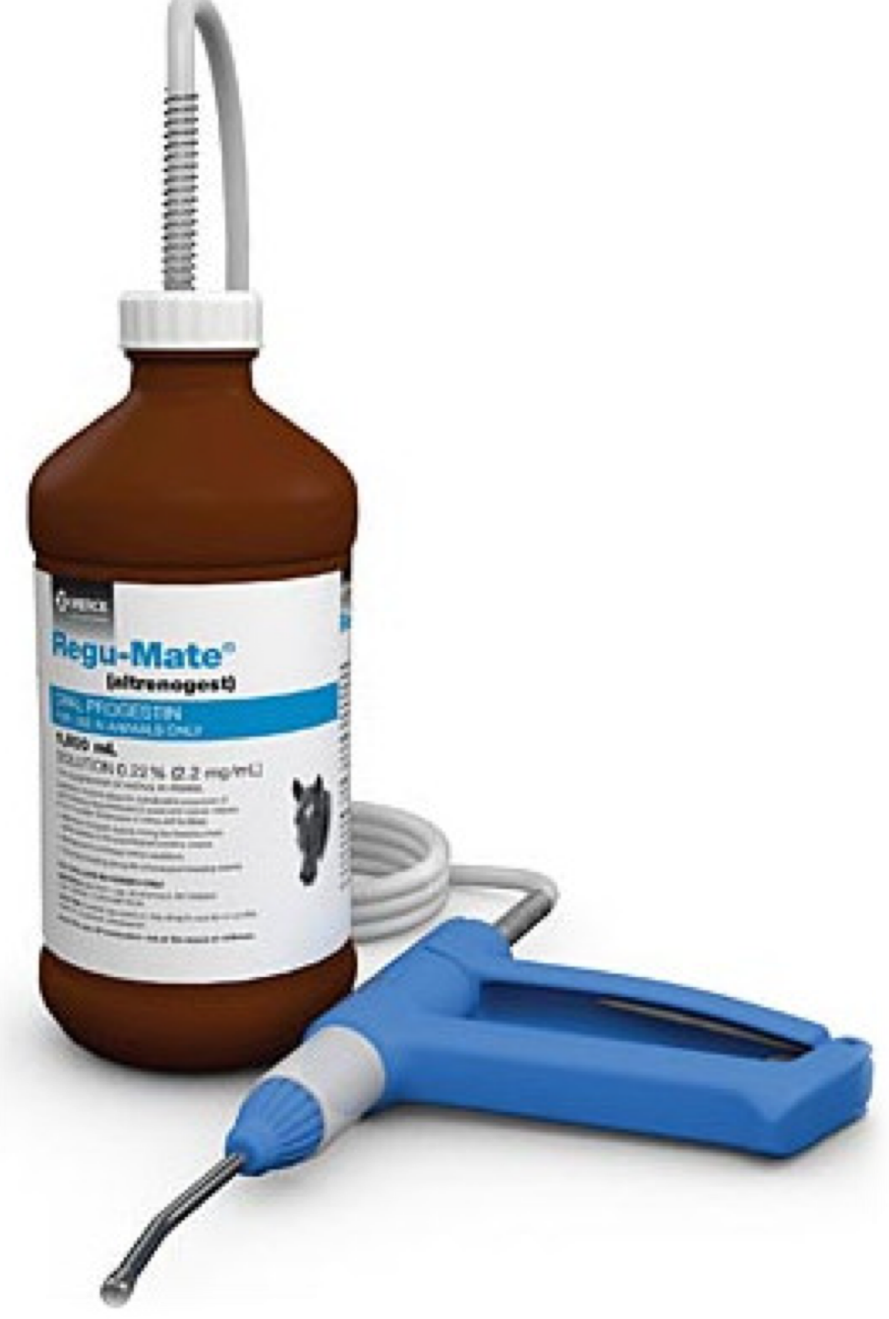




\section{Methods of Estrus Suppression}

- Administration of exogenous progesterone/progestins

- I.e. Oral Altrenogest

- Extending the functional span of the corpus luteum (CL)

- Intrauterine glass ball

- Oxytocin

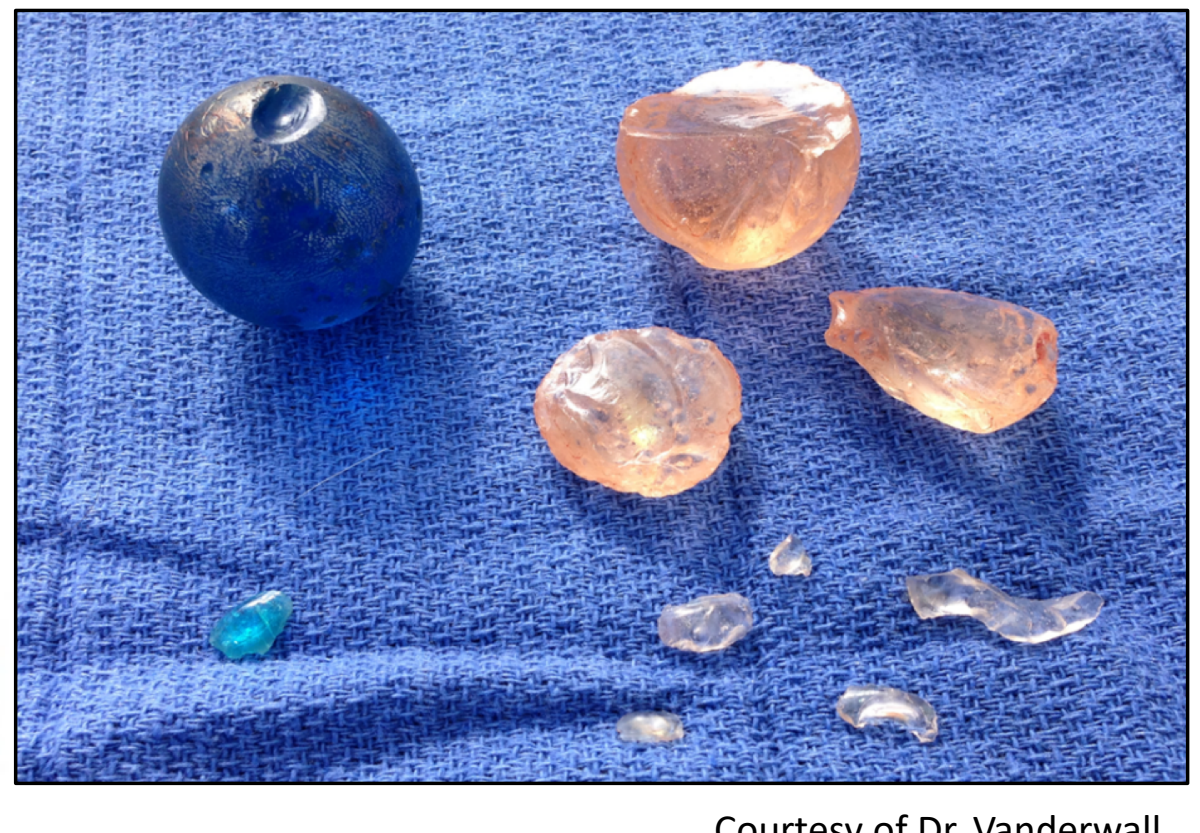

Courtesy of Dr. Vanderwall

hegu-Mate

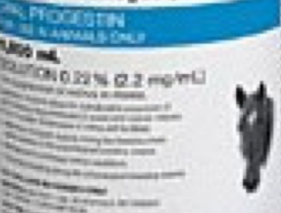




\section{Oxytocin}

- Released from the posterior pituitary gland

- Pulsatile nature

- Very short half-life

- Functions

- Milk let-down

- Stimulates uterine contractions

- Oxytocin-Prostaglandin luteolytic pathway

- Therapeutic use to prolong $\mathrm{CL}$ function

- 8-Day Protocol (1x daily: days 7-14)

- Slow-release Oxytocin (SR-OT: two treatments)

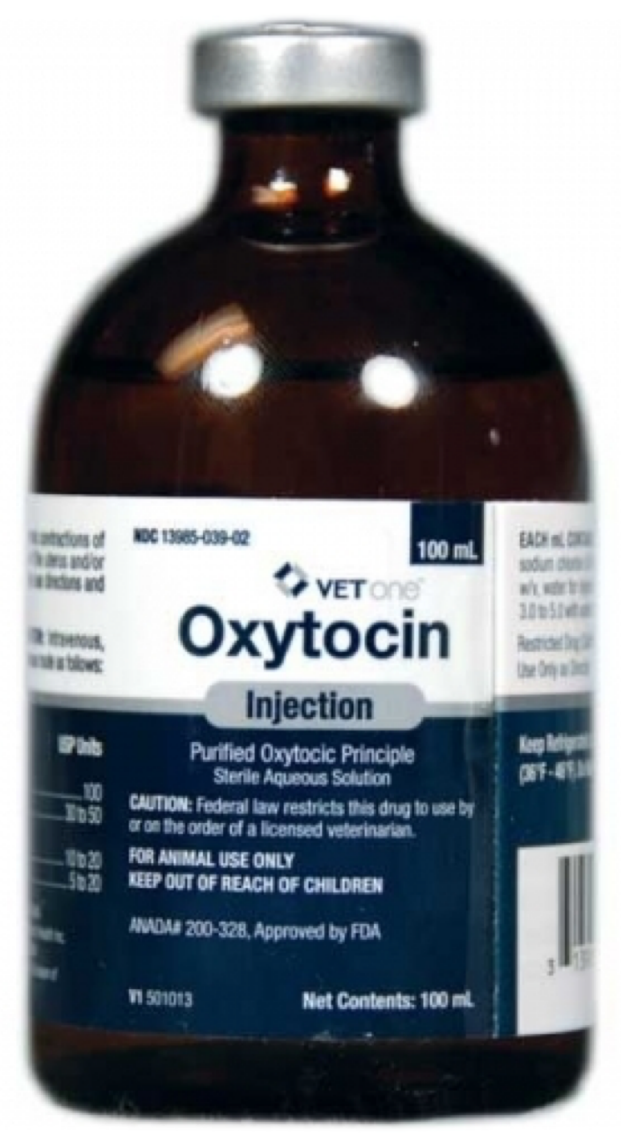




\section{Journal of Equine Veterinary Science}

Original Research

Evaluation of a Proprietary Slow-Release Oxytocin Formulation on Corpus Luteum Function in Mares

Brendan A. Sarnecky ${ }^{\mathrm{a}}$, Dirk K. Vanderwall ${ }^{\mathrm{a}, *}$, Holly M. Mason ${ }^{\mathrm{a}}$, Stephen M. Kirschner ${ }^{\mathrm{b}}$, Benson Ambrose ${ }^{\mathrm{a}}$,

Theda L. Parker ${ }^{a}$

a Department of Animal, Dairy and Veterinary Sciences, School of Veterinary Medicine, Utah State University, Logan, UT

${ }^{\mathrm{b}}$ Wildlife Pharmaceuticals, Inc, $R$ \& $D$ Department, Windsor, CO 


\section{Hypothesis}

- A two-injection proprietary SR-OT protocol will deliver an appropriate amount of oxytocin for a sufficient duration of time to inhibit luteolysis

\section{Objectives}

- Determine if IM administration of 2,400 IU of SR-OT once on days 7 and 10 after ovulation would prolong CL function in treated mares compared to a non-treated control group

- Reduce number of injections from previous aqueous oxytocin methods 


\section{Ovulation}

Detected via trans-rectal

ultrasound/palpation

ov

3 times per week

Until day 50

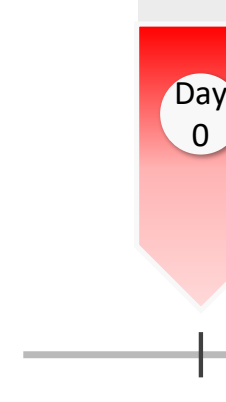

0
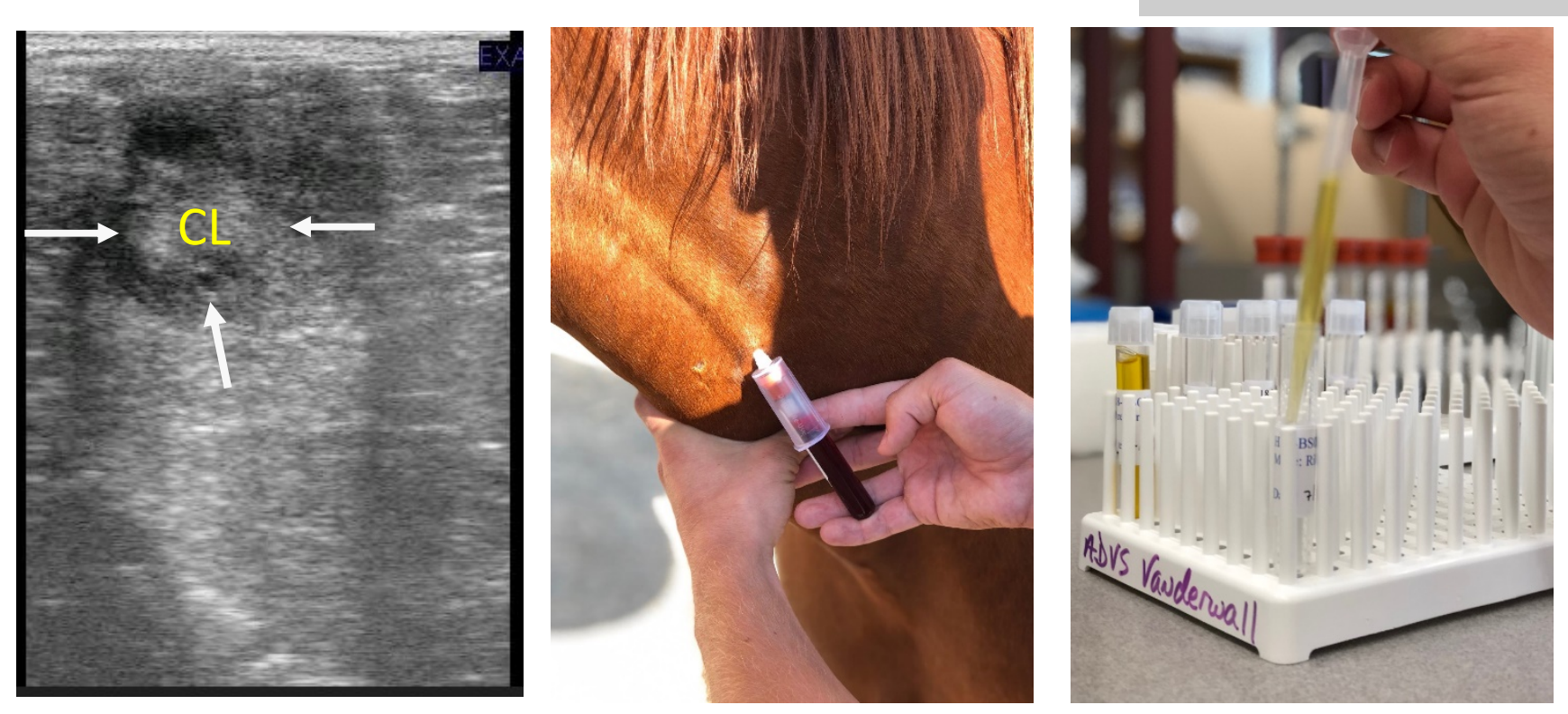

Day 10 SR-OT

2400 IU (1 CC)

Intramuscular

2400 IU (1 CC)

Intramuscular

\section{Groups \\ Control $n=8$ mares}

SR-OT Treatment $n=8$ mares

\section{UtahStateUniversity}

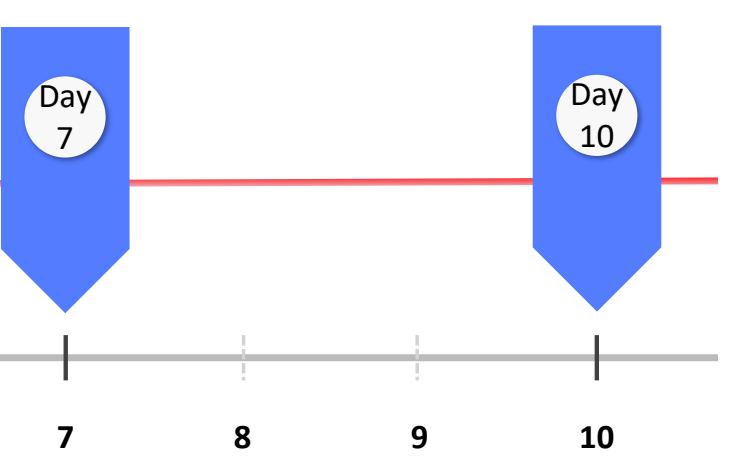

Days after ovulation

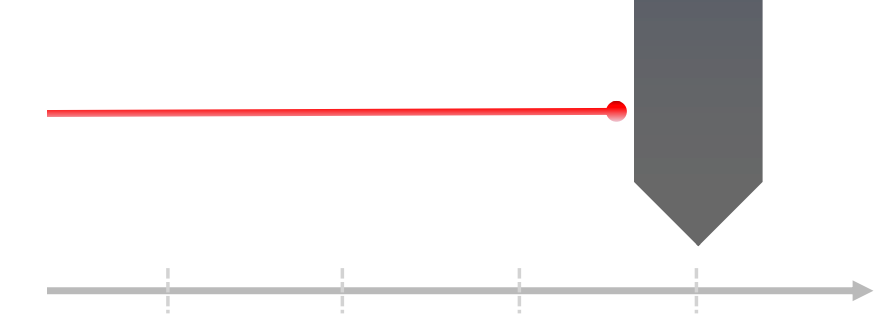

//

47

48

49

50

Progesterone

concentration evaluated

via chemiluminescent

enzyme immunoassay (Immulite Progesterone)

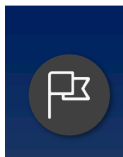




\section{Results}

- $0 / 8$ control mares with prolonged luteal function*

- $6 / 8$ treated mares with prolonged luteal function*
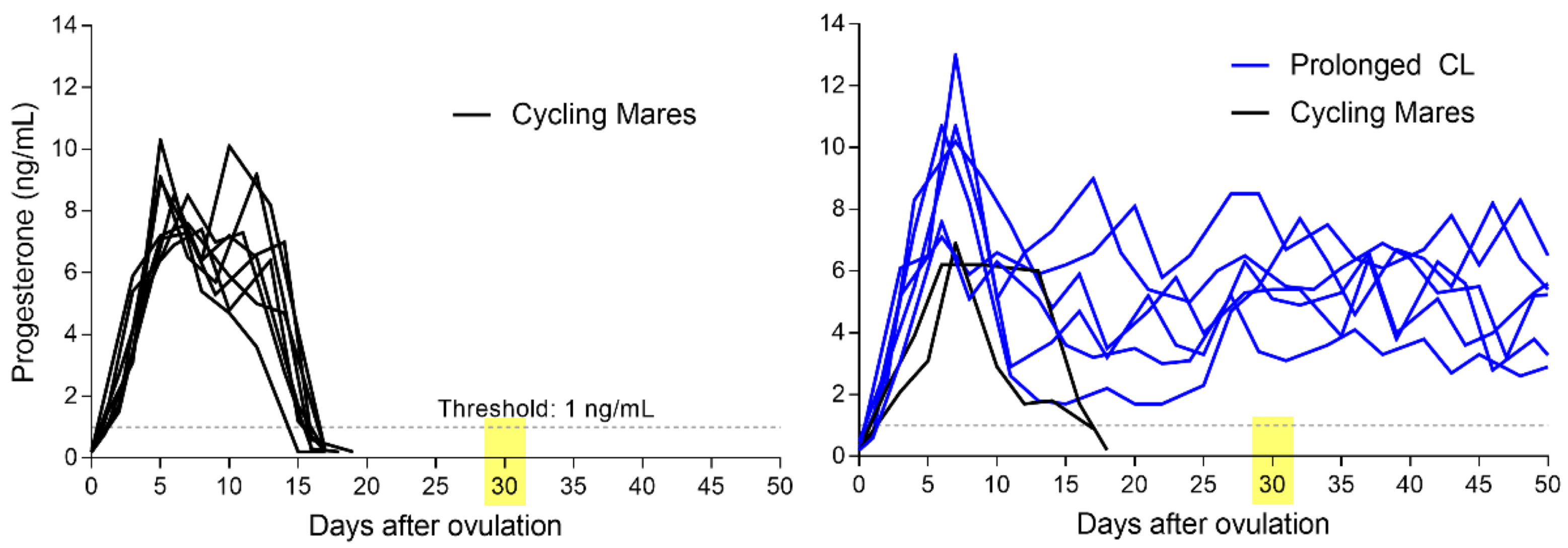

- Prolonged luteal function defined as $>1 \mathrm{ng} / \mathrm{ml}$ for over 30 days

- * Prolonged function compared with Fisher's exact test; $\mathrm{P}<.01$ 


\section{Conclusions}

- SR-OT administered on days 7 and 10 is an effective method of prolonging luteal function

- This proprietary SR-OT formulation provides a $75 \%$ reduction in number of treatments needed in previous aqueous oxytocin methods

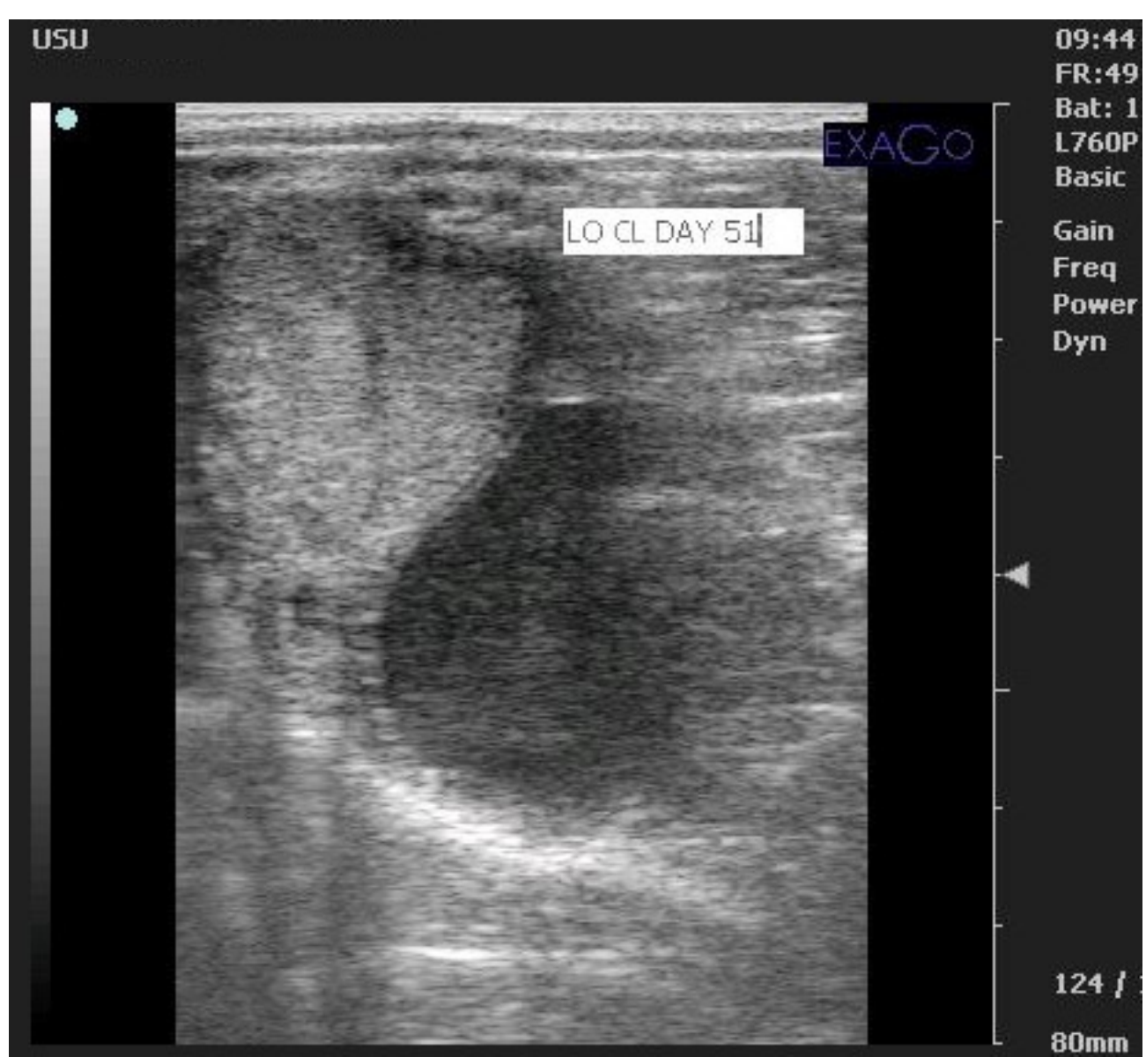




\section{Acknowledgements}

- Utah Agricultural Experiment Station (Project \# UTA01157)

- Wildlife Pharmaceuticals, Windsor, CO.

- JoAnna Buschmann, Ashlee Buist, Sherrie Petty, and Bettina Conrad 


\section{Conclusions}

- SR-OT administered on days 7 and 10 is an effective method of prolonging luteal function

- This proprietary SR-OT formulation provides a $75 \%$ reduction in number of treatments needed in previous aqueous oxytocin methods

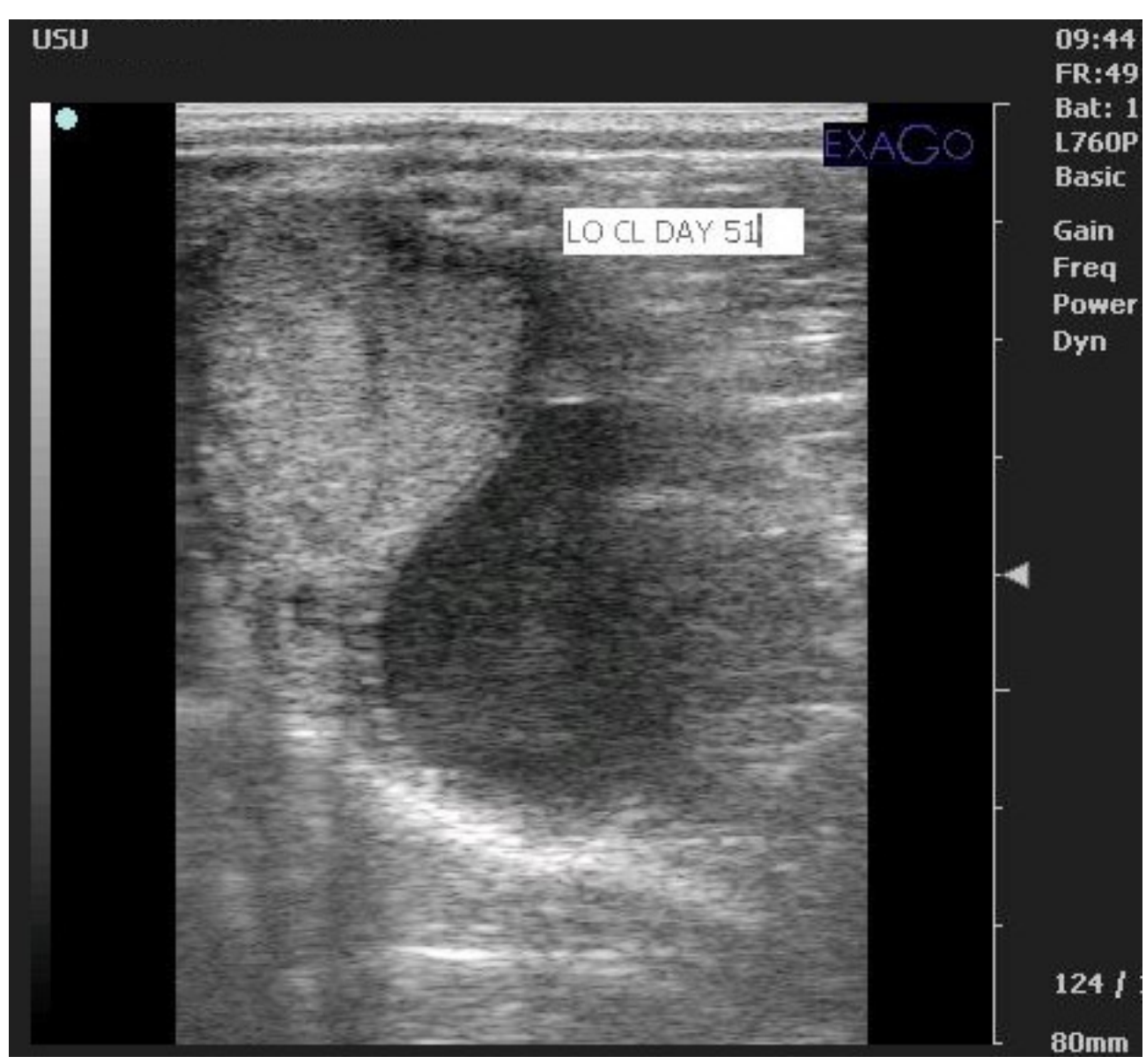




\section{Supporting Slides}




\section{Pro- vs Anti-Luteolytic Functions of Oxytocin}

\section{Equine Estrous Cycle}

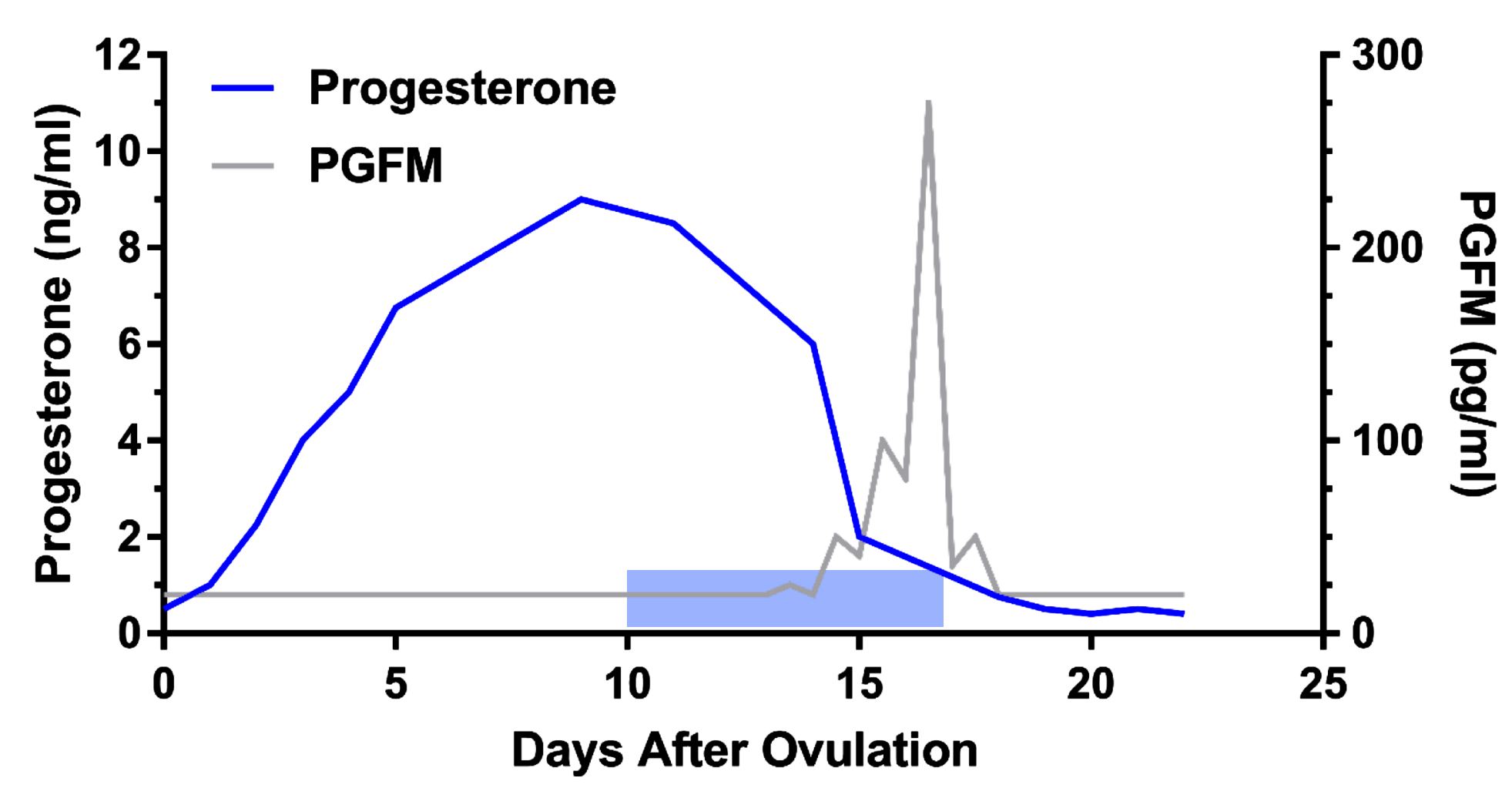

Pro-Luteolytic

- Oxytocin after day 10

Binds to oxytocin receptor at endometrial epithelium

Stimulates secondary messengers associated with PGF2 $\alpha$ synthesis

- COX II

PGF2 $\alpha$ circulates through the blood stream and targets the $\mathrm{CL}$

$\mathrm{CL}$ undergoes luteolysis 


\section{Pro- vs Anti-Luteolytic}

\section{Equine Estrous Cycle}

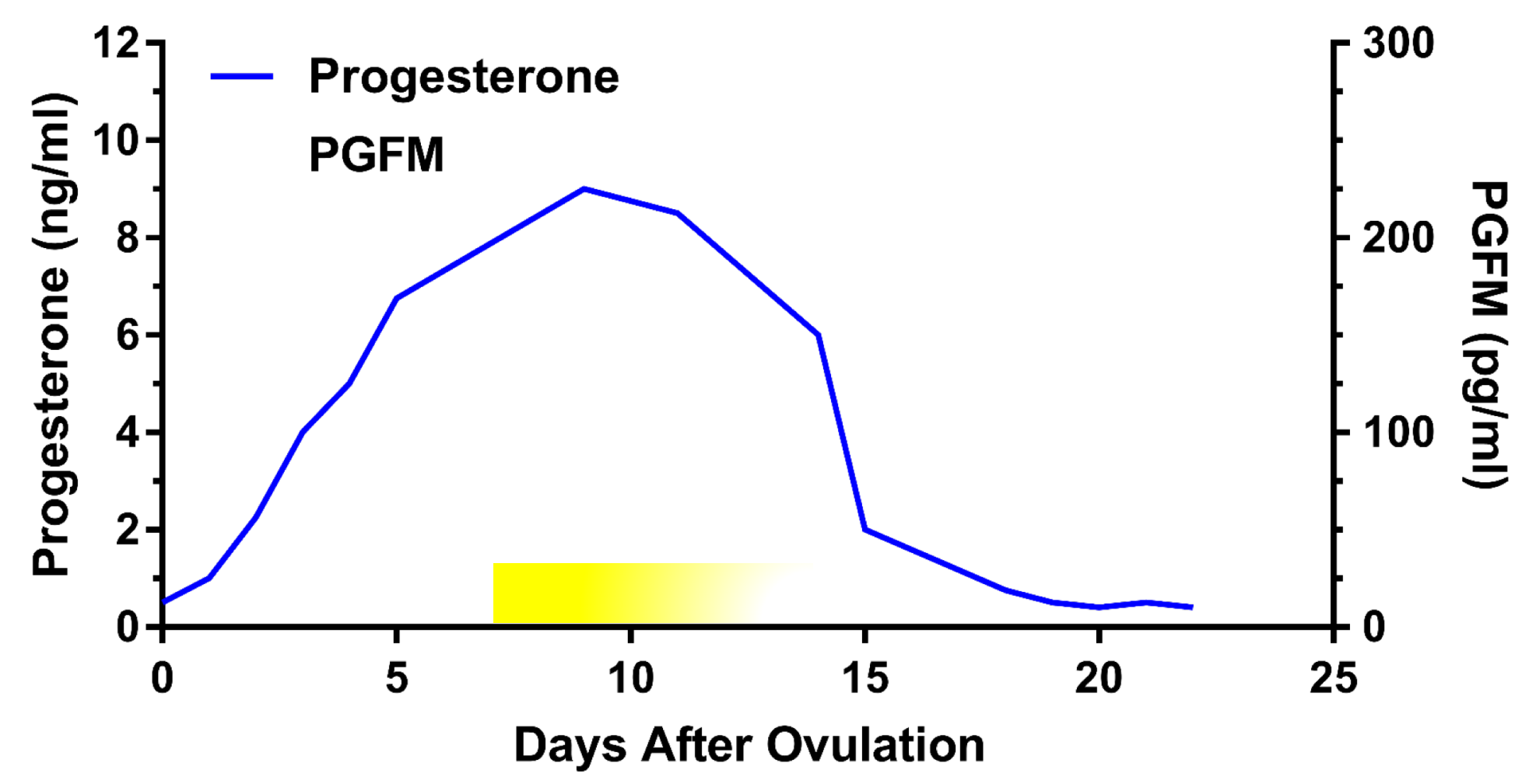

Anti-Luteolytic

- Oxytocin administered before day 10 and continued

- Binds to oxytocin receptor at endometrial epithelium

- Lack of secondary messenger

- Inhibits the upregulation of secondary messengers

- Specifically COX II

- No "spontaneous" luteolysis

- CL has prolonged function

- Up to 90 days 